\title{
WEIGHT STRINGS IN NONSTANDARD REPRESENTATIONS OF KAC-MOODY ALGEBRAS
}

\author{
MEIGHAN I. DILLON
}

\begin{abstract}
We consider the weights which occur in arbitrary irreducible highest weight representations of Kac-Moody algebras and determine conditions under which certain weights may or may not occur.
\end{abstract}

\section{INTRODUCTION}

Much has been written in recent years about the so-called standard or integrable highest weight representations of affine Lie algebras, the attention due in part to the intimate connections between these representations and the physicists string theories. (See $[1-3,6,8,10]$ for a sampling of the literature.) In this work we offer the slightly broader view of Lie algebra representations afforded by considering the class of irreducible highest weight representations of arbitrary Kac-Moody algebras, a class which includes the standard representations of affine Lie algebras and the irreducible finite-dimensional representations of finite-dimensional simple Lie algebras over $\mathbf{C}$.

The most fundamental object of our scrutiny is a Kac-Moody algebra $\mathfrak{g}$. Generally infinite-dimensional, $\mathfrak{g}$ is a complex Lie algebra defined by generators which satisfy relations as in $\S 1$. We point out here that underlying the relations satisfied by $\mathfrak{g}$ is an indecomposable $n \times n$ generalized Cartan matrix (GCM) $A$. (See [6, 7, 12-14].) If there is a column vector $u$ with components greater than zero $(u>0)$ such that $A u>0$, then $\mathfrak{g}$ is simple and finite dimensional. If there is $u>0$ such that $A u=0$ then $\mathfrak{g}$ is neither simple nor finite dimensional but is said to be affine. (See $[4,5]$ for the theory of finite-dimensional Lie algebras over $\mathbf{C}$; for the theory of affine Lie algebras, see $[6,7,11-14]$.) If $\mathfrak{g}$ is neither finite dimensional nor affine it is indefinite.

Our objective here is to describe the weights and the nature (finite or infinite) of the weight strings which occur in irreducible highest weight representations of $\mathfrak{g}$, an arbitrary Kac-Moody algebra. We give background on $\mathfrak{g}$ and its representations in $\S 1$. (All the material there may be found in [6].)

We start $\S 2$ with a technical result concerning the roots of $\mathfrak{g}$. We then fix a

Received by the editors September 14, 1988.

1980 Mathematics Subject Classification (1985 Revision). Primary 17B10, 17B65, 17 B67.

The material in this work was drawn partly from the author's Ph.D. thesis which was done under the supervision of John Faulkner at the University of Virginia. 
set of weight vectors in $\mathfrak{g}$ and go on to modify some of the terminology and results from $\S 1$ to fit the context of nonstandard representations of $\mathfrak{g}$.

In $\S 3$, we consider (nonstandard) irreducible highest weight representations of $\mathfrak{g}$ coming finally to our main result, Theorem 3.8 , where we describe the weight strings which occur in these modules.

\section{BACKGROUND}

Let $\mathfrak{g}$ be a Kac-Moody algebra, $e_{i}, f_{i}(i=1, \ldots, n)$ its Chevalley generators, $\Pi^{\mathrm{v}}=\left\{\alpha_{q}^{\mathrm{v}}, \ldots, \alpha_{1}^{\mathrm{v}}\right\}$ its simple coroots, $\Pi=\left\{\alpha_{1}, \ldots, \alpha_{n}\right\}$ its simple roots. Recall that $\Pi^{v}$ is linearly independent in the complex vector space $\mathfrak{h}^{*}$, the dual of $\mathfrak{h}$, the Cartan subalgebra of $\mathfrak{g}$. Letting $\langle\cdot, \cdot\rangle$ denote the pairing of $\mathfrak{h}$ and $\mathfrak{h}^{*}$, we have that $a_{i j}=\left\langle\alpha_{j}, \alpha_{i}^{\mathrm{v}}\right\rangle$ is a nonpositive integer when $i \neq j$ and that $a_{i i}=2$ for $i=1, \ldots, n$. Recall that $\mathfrak{g}$ satisfies

$$
\begin{gathered}
{\left[e_{i} f_{j}\right]=\delta_{i j} \alpha_{i}^{\mathrm{v}}, \quad\left[h e_{i}\right]=\left\langle\alpha_{i}, h\right\rangle e_{i},} \\
{\left[h f_{i}\right]=-\left\langle\alpha_{i}, h\right\rangle f_{i}, \quad\left[h h^{\prime}\right]=0,}
\end{gathered}
$$

ad $e_{i}^{-a_{i j}+1} e_{j}=0=\operatorname{ad} f_{i}^{-a_{i j}+1} f_{j}$ for $h, h^{\prime}$ in $\mathfrak{h}$ and $i, j=1, \ldots, n$. If we let $\mathfrak{g}_{\left(\alpha_{i}\right)}:=\mathbf{C} e_{i}+\mathbf{C} \alpha_{i}^{\mathrm{v}}+\mathbf{C} f_{i}$ we see that $\mathfrak{g}_{\left(\alpha_{i}\right)} \cong \mathbf{s l}_{2}(\mathbf{C})$ for $i=1, \ldots, n$.

Denote by $\omega$ the involutive automorphism of $\mathfrak{g}$ determined by $\omega\left(e_{i}\right)=-f_{i}$, $\omega\left(f_{i}\right)=-e_{i}, \omega(h)=-h$ for $h$ in $\mathfrak{h}, i=1, \ldots, n$. We call $\omega$ the Cartan involution of $\mathfrak{g}$.

If $\left[\cdots\left[e_{i_{1}} e_{i_{2}}\right] \cdots e_{i_{m}}\right]=\left[e_{i_{1}} \cdots e_{i_{m}}\right]$ is nonzero, then using the Cartan involution we see that $\left[f_{i_{1}} \cdots f_{i_{m}}\right]$ is also nonzero. In this case, $\alpha=\sum_{j=1}^{m} \alpha_{i_{j}}$ is called a positive root of $\mathfrak{g},-\alpha$, a negative root. We denote the set of roots of $\mathfrak{g}$ by $\Delta$, the positive roots by $\Delta_{+}$, the negative roots by $\Delta_{-}$. Recall that $\mathfrak{g}$ has the root space decomposition $\mathfrak{g}=\bigoplus_{\alpha \in \Delta \cup\{0\}} \mathfrak{g}_{\alpha}$ where $\mathfrak{g}_{\alpha}=\{x \in \mathfrak{g}:[h x]=\langle\alpha, h\rangle x$ for all $h \in \mathfrak{h}\}$ and $\mathfrak{g}_{0}=\mathfrak{h}$.

Let $\mathfrak{n}_{+}=\bigoplus_{\alpha \in \Delta_{+}} \mathfrak{g}_{\alpha}, \mathfrak{n}_{-}=\bigoplus_{\alpha \in \Delta_{-}} \mathfrak{g}_{\alpha}$. If $U(\mathfrak{g})$ is the universal enveloping algebra of $\mathfrak{g}$, the PBW theorem $($ see $[4,5])$ give us that $U(\mathfrak{g})=U\left(\mathfrak{n}_{-}\right) \otimes U(\mathfrak{g}) \otimes$ $U\left(\mathfrak{g}_{+}\right)$.

If $\alpha$ is any element in the $\mathbf{Z}_{+}$span of $\Pi$, then we define the height of $\alpha$, denoted ht $\alpha$, by ht $\alpha=\sum_{i}^{n} k_{i}$ where $\alpha=\sum_{1}^{n} k_{i} \alpha_{i}$. The support of $\alpha$, denoted $\operatorname{supp} \alpha$, is the set of $\alpha_{i}$ in $\Pi$ such that $k_{i} \neq 0$ again when $\alpha=\sum_{1}^{n} k_{i} \alpha_{i}$.

Each $\alpha_{i}$ in $\Pi$ determines $r_{i}$, the fundamental reflection on $\mathfrak{h}^{*}$, given by $r_{i}(\lambda)=\lambda-\left\langle\lambda, \alpha_{i}^{\mathrm{v}}\right\rangle \alpha_{i}$ for $\lambda$ in $\mathfrak{h}^{*}$. The group $W$ which is generated by these reflections is called the Weyl group. We use $W$ to classify the roots of $\mathfrak{g}$ as follows.

If $\alpha$ in $\Delta$ has the property that there is $w$ in $W$ such that $w(\alpha)$ belongs to $\Pi$, then $\alpha$ is a real root, otherwise, $\alpha$ is an imaginary root. We denote the real and imaginary roots of $\mathfrak{g}$ by $\Delta^{\mathrm{re}}$ and $\Delta^{\mathrm{im}}$ respectively. Note that if $\alpha$ belongs to $\Delta^{\text {re }}$, then $\operatorname{dim} \mathfrak{g}_{\alpha}=1$; if $\alpha$ belongs to $\Delta^{\text {im }}$, then $\infty>\operatorname{dim} \mathfrak{g}_{\alpha} \geq 1$. 
Just as $r_{i}$ is defined on $\mathfrak{h}^{*}$ for $i=1, \ldots, n$ we may define the reflection determined by $\alpha_{i}^{\mathrm{v}}$ on $\mathfrak{h}$ via $r_{i}(h)=h-\left\langle h, \alpha_{i}\right\rangle \alpha_{i}^{\mathrm{v}}$. The group generated by these reflections may be identified with $W$. If $\alpha$ belongs to $\Delta^{\mathrm{re}}$ and $w\left(\alpha_{i}\right)=\alpha$ for some $w$ in $W, \alpha_{i}$ in $\Pi$, we define $\alpha^{\mathrm{v}}$ in $\mathfrak{h}$ via $w\left(\alpha_{i}^{\mathrm{v}}\right)=\alpha^{\mathrm{v}}$. We then have $r_{\alpha}$ in $W$ defined for all $\alpha$ in $\Delta^{\text {re }}$ via $r_{\alpha}(\lambda)=\lambda-\left\langle\lambda, \alpha^{\mathrm{v}}\right\rangle \alpha$ for $\lambda$ in $\mathfrak{h}^{*}$.

Suppose now that $\pi: \mathfrak{g} \rightarrow \operatorname{End}_{c}(V)$ is a representation of $\mathfrak{g}$ such that $V=$ $\bigoplus_{\lambda \in \mathfrak{h}^{*}} V_{\lambda}$, where $V_{\lambda}=\{v \in V: h \cdot v=\langle\lambda, h\rangle v$ for all $h \in \mathfrak{h}\} . V$ is then $\mathfrak{h}$ diagonalizable and the set of all $\lambda$ in $\mathfrak{h}^{*}$ such that $V_{\lambda} \neq 0$ is the set of weights of $V$. (Note that under the adjoint action, $\mathfrak{g}$ is itself an $\mathfrak{h}$-diagonalizable $\mathfrak{g}$ module.) The multiplicity of a weight $\lambda$, denoted mult $\lambda$, is the dimension of $V_{\lambda}$.

If $x$ in $\mathfrak{g}$ has the property that for each $v$ in $V$ there is $N$ in $\mathbf{Z}_{+}$such that $x^{N} \cdot v=0$ then $x$ is said to be locally nilpotent on $V$. If all $e_{i}$ and $f_{i}(i=1, \ldots, n)$ are locally nilpotent on an $\mathfrak{h}$-diagonalizable $\mathfrak{g}$ module then the representation is said to be integrable or standard. (Note that $U(\mathfrak{g})$ is an integrable $\mathfrak{g}$ module under the adjoint action of $\mathfrak{h}$.)

If $V$ is an integrable $\mathfrak{g}$ module we define automorphisms on $V$ by $r_{i}^{\pi}=$ $\exp \pi\left(e_{i}\right) \exp \pi\left(-f_{i}\right) \exp \pi\left(e_{i}\right), i=1, \ldots, n$. Then for $x_{1}, \ldots, x_{m}$ in $\mathfrak{g}, v$ in $V($ see $[16$, Lemma 14])

$$
r_{i}^{\pi}\left(x_{1} \cdots x_{m} \cdot v\right)=r_{i}^{\mathrm{ad}}\left(x_{1}\right) \cdots r_{i}^{\mathrm{ad}}\left(x_{m}\right) \cdot r_{i}^{\pi}(v) .
$$

Applying the representation theory of $\operatorname{sl}_{2}(\mathbf{C}) \cong \mathfrak{g}_{\left(\alpha_{i}\right)}$ to $V$ we get (see [6, Proposition 3.6])

Proposition 1.2 (Kac). If $V$ is an integrable $\mathfrak{g}$ module then

(a) $V$ decomposes into a direct sum of finite-dimensional $\mathfrak{g}_{\left(\alpha_{i}\right)}$ modules;

(b) if $\lambda$ is a weight of $V$, then

(i) $r_{i}^{\pi}\left(V_{\lambda}\right)=V_{r_{i}(\lambda)}$;

(ii) $\lambda+t \alpha_{i}$ is a weight of $V$ if and only if $t$ is an integer lying between $-p$ and $q$ where $p=q=\infty$ or $p$ and $q$ are fixed nonnegative integers which satisfy $\left\langle\lambda, \alpha_{i}^{\mathrm{v}}\right\rangle=p-q$

(iii) $e_{i} \cdot V_{\lambda} \neq 0$ if $\lambda+\alpha_{i}$ is a weight;

(iv) $\left\langle\lambda, \alpha_{i}^{\mathrm{v}}\right\rangle \geq 0$ (respectively $\left.\left\langle\lambda, \alpha_{i}^{\mathrm{v}}\right\rangle \leq 0\right)$ if $\lambda+\alpha_{i}$ (respectively $\left.\lambda-\alpha_{i}\right)$ is not a weight.

If $\lambda$ is a weight of $V$ and $\alpha$ is in $\Delta$ then the $\alpha$-string through $\lambda$ is the set of all weights of the form $\lambda+t \alpha$ where $t$ is an integer.

Suppose now that $V$ is a highest weight module, i.e., that there is $v^{+}$in $V$ such that $\mathfrak{n}_{+} \cdot v^{+}=0, h \cdot v^{+}=\langle\Lambda, h\rangle v^{+}$for some $\Lambda$ in $\mathfrak{h}^{*}$ for all $h$ in $\mathfrak{h}$, and that $V=U\left(\mathfrak{n}_{-}\right) \cdot v^{+}$. We call $v^{+}$a highest weight vector and $\Lambda$ the highest weight of $V$. Note that $V$ is $\mathfrak{h}$-diagonalizable and that for all weights $\lambda$ of $V$, mult $\lambda<\infty$. If $V$ is, in addition, freely generated as a $U\left(\mathfrak{n}_{-}\right)$module, then $V$ has a unique maximal proper submodule $V^{\prime}$. Letting $M(\Lambda)=V / V^{\prime}$, 
we get the (unique) irreducible $\mathfrak{g}$ module with highest weight $\Lambda . M=M(\Lambda)$ will be the focus of our attention in the sequel.

\section{Preliminaries}

We need a technical result before undertaking a study of the representations of $\mathfrak{g}$.

Lemma 2.1. If $\alpha$ is in $\Delta_{+}$and $\alpha_{k}$ belongs to $\operatorname{supp} \alpha$, then $\alpha$ may be written in the form $\alpha=\alpha_{k}+\sum_{j=1}^{m} \alpha_{i_{j}}$ where $\alpha_{i_{j}}$ belongs to $\Pi$ and for all $t$ in $\{1, \ldots, m\}$, $\alpha_{k}+\sum_{j=1}^{t} \alpha_{i_{j}}$ belongs to $\Delta_{+}$.

Proof. We proceed by induction on ht $\alpha$. If $\alpha$ is in $\Pi$ the result is trivial so assume that ht $\alpha>1$. Pick $\alpha_{1}$ in $\operatorname{supp} \alpha$ so that

$$
\beta=\alpha-\alpha_{1} \in \Delta_{+} \text {. }
$$

Since ht $\beta<$ ht $\alpha$, it follows by induction on ht $\alpha$ that the result is true for $\beta$, hence for $\alpha$ in the event that $\operatorname{supp} \alpha=\operatorname{supp} \beta$. Suppose then that $\operatorname{supp} \alpha \neq$ $\operatorname{supp} \beta$, i.e., that $\alpha_{1}$ is not in $\operatorname{supp} \beta$. Since $\alpha=\beta+\alpha_{1}$ it suffices to show that $\alpha=\alpha_{1}+\sum_{j=1}^{m} \alpha_{i_{j}}$ where $\alpha_{1}+\sum_{j=1}^{t} \alpha_{i_{j}}$ is in $\Delta_{+}$for $t=1, \ldots, m$, for some sequence of elements $\alpha_{i_{j}}$ in $\Pi$. Proposition 1.2 gives us that $\left\langle\beta, \alpha_{1}^{\mathrm{v}}\right\rangle \leq 0$ since $\beta+\alpha_{1}$ is in $\Delta_{+}$. Since $\beta-\alpha_{1}$ is not in $\Delta_{+}$, Proposition 1.2 also gives us that $\left\langle\beta, \alpha_{1}^{\mathrm{v}}\right\rangle<0$; in particular, there is $\alpha_{i_{1}}$ in $\operatorname{supp} \beta$ such that $\left\langle\alpha_{i_{1}}, \alpha_{1}^{\mathrm{v}}\right\rangle<0$ implying that $\alpha_{i_{1}}+\alpha_{1}$ belongs to $\Delta_{+}$. Again since ht $\beta<\mathrm{ht} \alpha$ we have by induction that $\beta=\alpha_{i_{1}}+\sum_{k=2}^{m} \alpha_{i_{j}}$ where $\alpha_{i_{j}}$ is in $\Pi$ for $j=2, \ldots, m$ and $\sum_{j=1}^{t} \alpha_{i_{j}}$ is in $\Delta_{+}$for $t=2, \ldots, m$. Since $\alpha_{1}$ is not in $\operatorname{supp} \beta$ we have for $j=2, \ldots, m\left\langle\alpha_{i_{j}}, \alpha_{1}^{\mathrm{v}}\right\rangle \leq 0$ thus, that $\left\langle\sum_{j=1}^{t} \alpha_{i_{j}}, \alpha_{1}^{\mathrm{v}}\right\rangle<0$ for $t=2, \ldots, m$. We conclude that $\alpha_{1}+\sum_{j=1}^{t} \alpha_{i_{j}}$ belongs to $\Delta_{+}$for $t=2, \ldots, m$ as desired.

For convenience, we shall fix a set of root vectors of $\mathfrak{g}$. It turns out that if $\alpha$ is real, $\left[\mathfrak{g}_{\alpha} \mathfrak{g}_{-\alpha}\right]=\mathbf{C} \alpha^{\mathrm{v}}$. Given any positive real root $\alpha$, we may thus pick $e_{\alpha}$ in $\mathfrak{g}_{\alpha}$ and $f_{\alpha}$ in $\mathfrak{g}_{-\alpha}$ such that $\left[e_{\alpha} f_{\alpha}\right]=\alpha^{\mathrm{v}}$. Since $\left\langle\alpha, \alpha^{\mathrm{v}}\right\rangle=2$ we have that $\mathfrak{g}_{(\alpha)}:=\mathbf{C} e_{\alpha}+\mathbf{C} \alpha^{\mathrm{v}}+\mathbf{C} f_{\alpha}$ is isomorphic to $\operatorname{sl}_{2}(\mathbf{C})$ for all $\alpha$ in $\Delta_{+}^{\mathrm{re}}$. We shall exploit this extensively below. If $\alpha$ is an arbitrary positive root, we shall use $x_{\alpha}$ and $x_{-\alpha}$ to designate nonzero elements of $\mathfrak{g}_{\alpha}$ and $\mathfrak{g}_{-\alpha}$ respectively.

Let $\pi: \mathfrak{g} \rightarrow \operatorname{End}_{\mathbf{C}}(V)$ be an $\mathfrak{h}$-diagonalizable representation of $\mathfrak{g}$. If $V$ is not necessarily integrable we say that the representation is nonstandard or nonintegrable. If $\alpha$ is a positive root with the property that all $x_{\alpha}$ and $x_{-\alpha}$ are locally nilpotent on $V$ then $\alpha$ is an integrable root relative to $V$. If $\alpha$ is real and integrable we define an automorphism on $V \quad r_{\alpha}^{\pi}=\exp \pi\left(e_{\alpha}\right) \exp \pi\left(-f_{\alpha}\right) \exp \pi\left(e_{\alpha}\right)$. As in (1.1) $r_{\alpha}^{\pi}\left(x_{1} \cdots x_{m} \cdot v\right)=r_{\alpha}^{\mathrm{ad}}\left(x_{1}\right) \cdots r_{\alpha}^{\mathrm{ad}}\left(x_{m}\right) \cdot r_{\alpha}^{\pi}(v)$ when $x_{1}, \ldots, x_{m}$ belong to $\mathfrak{g}, v$ to $V$. If a positive root $\alpha$ is not integrable, we say that $\alpha$ is a nonintegrable root. Using the representation theory of $\operatorname{sl}_{2}(\mathbf{C}) \cong \mathfrak{g}_{(\alpha)}$ for $\alpha$ real integrable, we generalize Proposition 1.2 as follows. 
Proposition 2.2. Let $V$ be an $\mathfrak{h}$-diagonalizable $\mathfrak{g}$ module and $\alpha$ a real integrable root relative to $V$. Then

(a) $V$ decomposes into a direct sum of finite-dimensional irreducible $\mathfrak{g}_{(\alpha)}$ modules;

(b) if $\lambda$ is a weight of $V$, then

(i) $r_{\alpha}^{\pi}\left(V_{\lambda}\right)=V_{r_{\alpha}(\lambda)}$;

(ii) $\lambda+t \alpha$ is a weight of $V$ if and only if $t$ is an integer lying between $-p$ and $q$ where $p=q=\infty$ or $p$ and $q$ are fixed nonnegative integers which satisfy $\left\langle\lambda, \alpha^{\mathrm{v}}\right\rangle=p-q$

(iii) $e_{\alpha} \cdot V_{\lambda} \neq 0$ if $\lambda+\alpha$ is a weight;

(iv) $\left\langle\lambda, \alpha^{\mathrm{v}}\right\rangle \geq 0$ (respectively $\left\langle\lambda, \alpha^{\mathrm{v}}\right\rangle \leq 0$ ) if $\lambda+\alpha$ (respectively $\lambda-\alpha$ ) is not a weight.

We close this section with a technical lemma.

Lemma 2.3. Let $V$ be an $\mathfrak{h}$-diagonalizable $\mathfrak{g}$ module and $\lambda$ a weight of $V$. If $v$ is an element of $V_{\lambda}$ then for all $\alpha$ in $\Delta_{+}^{\mathrm{re}}$ and $N$ in $\mathbf{Z}_{+}$,

$$
e_{\alpha} \cdot f_{\alpha}^{N} \cdot v=N\left(\left\langle\lambda, \alpha^{\mathrm{v}}\right\rangle-(N-1)\right) f_{\alpha}^{N} \cdot v+f_{\alpha}^{N} \cdot e_{\alpha} \cdot v
$$

Proof. See [7, §7.2].

\section{WEIGHTS IN NONSTANDARD MODULES}

Let $M$ be an irreducible highest weight module over $\mathfrak{g}$ with highest weight $\Lambda$ in $\mathfrak{h}^{*}$. Let $v^{+}$be a highest weight vector of $M$ and $M=\bigoplus_{\lambda \leq \Lambda} M_{\lambda}$ its weight space decomposition. We make no assumptions as to the integrability of $M$ but do assume that the representation is faithful. Our first goal is to determine which positive roots of $\mathfrak{g}$ are integrable.

Let $\Pi(\Lambda)=\left\{\alpha_{i} \in \Pi:\left\langle\Lambda, \alpha_{i}^{\mathrm{v}}\right\rangle \in \mathbf{Z}_{+}\right\}$.

Lemma 3.1. $f_{i}$ is locally nilpotent on $M$ if and only if $\alpha_{i}$ belongs to $\Pi(\Lambda)$. Proof. By Lemma 2.3,

$$
e_{i} \cdot f_{i}^{N} \cdot v^{+}=N\left(\left\langle\Lambda, \alpha_{i}^{\mathrm{v}}\right\rangle-(N-1)\right) f_{i}^{n-1} \cdot v^{+}
$$

for all $N$ in $\mathbf{Z}_{+}$. If $\alpha_{i}$ is not in $\Pi(\Lambda)$, it follows that $e_{i} \cdot f_{i}^{N} \cdot v^{+}$is nonzero for all positive integers $N$, thus, that $f_{i}^{N} \cdot v^{+}$is nonzero.

If $\alpha_{i}$ is in $\Pi(\Lambda)$, let $N=\left\langle\Lambda, \alpha_{i}^{\mathrm{v}}\right\rangle+1$. Then $e_{j} \cdot f_{i}^{N} \cdot v^{+}=0$ for $j=1, \ldots, n$ implying by $[6, \S 9.3]$ that $f_{i}^{N} \cdot v^{+}=0$. Since $v^{+}$generates $M$, the result follows by [6, Lemma 3.4].

If $\alpha$ belongs to $\Delta^{\text {re }}$, let $W_{\alpha}$ be the subgroup of $W$ generated by the set $\left\{r_{i}: \alpha_{i} \in \operatorname{supp} \alpha\right\}$.

Lemma 3.2. If $\alpha$ is in $\Delta^{\mathrm{re}}$ there is $w$ in $W_{\alpha}$ and $\alpha_{j}$ in $\operatorname{supp} \alpha$ such that $\alpha=w\left(\alpha_{j}\right)$. 
Proof. If $\alpha>0$ and ht $\alpha>1$ then by [6, Lemma 5.4] there is $\alpha_{j}$ in $\Pi$ such that $\left\langle\alpha, \alpha_{j}^{\mathrm{v}}\right\rangle>0$. We then know that $r_{j}(\alpha)=\alpha-\left\langle\alpha, \alpha_{j}^{\mathrm{v}}\right\rangle \alpha_{j}$ is a positive real root. Since $\operatorname{ht}\left(r_{j}(\alpha)\right)<\operatorname{ht}(\alpha)$ the result follows in this case by induction on ht $\alpha$.

If $\alpha<0,-\alpha$ is in $\Delta_{+}^{\text {re }}$ so there is $w$ in $W_{-\alpha}=W_{\alpha}$ and $\alpha_{j}$ in $\operatorname{supp}(-\alpha)=$ $\operatorname{supp} \alpha$ such that $-\alpha=w\left(\alpha_{j}\right)$. We conclude that $\alpha=w\left(-\alpha_{j}\right)=w\left(r_{j}\left(\alpha_{j}\right)\right)$ as desired.

Let $\Delta_{+}(\Lambda)=\left\{\alpha \in \Delta_{+}: \operatorname{supp} \alpha \subset \Pi(\Lambda)\right\}$.

Lemma 3.3. If $\alpha$ belongs to $\Delta^{\mathrm{re}} \cap \Delta_{+}(\Lambda)$, then $f_{\alpha}$ is locally nilpotent on $M$.

Proof. Let $\lambda$ be a weight of $M$ and $\alpha$ an element of $\Delta^{\mathrm{re}} \cap \Delta_{+}(\Lambda)$. For all $\alpha_{i}$ in $\operatorname{supp} \alpha, r_{i}^{\pi}\left(M_{\lambda}\right)=M_{r_{i}(\lambda)}$ so that if $w=r_{i_{k}} \cdots r_{i_{1}}$ belongs to $W_{\alpha}$ where $\alpha_{i_{j}}$ is in $\operatorname{supp} \alpha, j=1, \ldots, k$, then $w^{\pi}\left(M_{\lambda}\right)=M_{w(\lambda)}$.

Invoking Lemma 3.2, we choose $w$ in $W_{\alpha}$ so that $w\left(\alpha_{i}\right)=\alpha$ for some $\alpha_{i}$ in $\operatorname{supp} \alpha$. Since $\alpha_{i}$ is integrable, there is $N$ in $\mathbf{Z}_{+}$such that $M_{\Lambda-N \alpha_{i}}=0$. Let $\lambda=w^{-1}(\Lambda)$. Then $w^{\pi}\left(M_{\lambda-N \alpha_{i}}\right)=M_{w\left(\lambda-N \alpha_{i}\right)}=M_{\Lambda-N \alpha}=0$ implying in particular that $f_{\alpha}^{N} \cdot M_{\Lambda}=0$. By [6, Lemma 3.4], we conclude that $f_{\alpha}$ is locally nilpotent on $M$.

The following lemma ${ }^{1}$ will facilitate our study of nonintegrable roots.

Lemma 3.4. If $\alpha$ is in $\Delta_{+}^{\mathrm{re}}$ and $f_{\alpha}^{N} \cdot v=0$ for some nonzero $v$ in $M, N$ in $\mathbf{Z}_{+}$, then $f_{\alpha}$ is locally nilpotent on $M$.

Proof. Without loss of generality we may assume that $f_{\alpha}^{N-1} \cdot v \neq 0$. The irreducibility of $M$ then implies that $M=U(\mathfrak{g}) \cdot f_{\alpha}^{N-1} \cdot v$. Given $u$ in $M$, there is then $g$ in $U(\mathfrak{g})$ so that $u=g \cdot f_{\alpha}^{N-1} \cdot v$. Noting that

$$
f_{\alpha} \cdot u=f_{\alpha} \cdot g \cdot f_{\alpha}^{N-1} \cdot v=\left[f_{\alpha} g\right] \cdot f_{\alpha}^{N-1} \cdot v+g \cdot f_{\alpha}^{N} \cdot v=\operatorname{ad} f_{\alpha} \cdot g \cdot f_{\alpha}^{N-1} \cdot v
$$

we see that $f_{\alpha}^{r} \cdot u=\left(\operatorname{ad} f_{\alpha}\right)^{r} \cdot g \cdot f^{N-1} \cdot v$. As $f_{\alpha}$ is locally nilpotent on $U(\mathfrak{g})$ under the adjoint action, we may choose $r$ large enough so that $\left(\operatorname{ad} f_{\alpha}\right)^{r} \cdot g=0$, hence, $f_{\alpha}^{r} \cdot u=0$ proving the lemma.

Our first result concerning nonintegrable roots is now obvious.

Lemma 3.5. If $\alpha_{j}$ belongs to $\Pi \backslash \Pi(\Lambda)$, then $f_{j} \cdot v$ is nonzero for all nonzero $v$ in $M$.

Proof. The result is an immediate consequence of Lemmas 3.1 and 3.4.

Recall that if $\mathfrak{g}$ is affine it has a minimal positive imaginary root $\delta$ with the property that $\left\langle\delta, \alpha_{i}^{\mathrm{v}}\right\rangle=0$ for all $\alpha_{i}^{\mathrm{v}}$ in $\Pi^{\mathrm{v}}$. If $\beta$ belongs to $\Delta^{\mathrm{im}}$, then $\beta=r \delta$ for some integer $r$. The imaginary roots of an affine Lie algebra in an irreducible highest weight representation behave in a singular fashion as the next result shows.

\footnotetext{
${ }^{1}$ I would like to thank the referee who pointed this out to me.
} 
Lemma 3.6. Suppose $\mathfrak{g}$ is affine, $\beta$ is in $\Delta_{+}^{\mathrm{im}}$, and $x_{-\beta}$ is a nonzero element of $\mathfrak{g}_{-\beta}$. For all weights $\lambda$ of $M, \lambda-\beta$ is also a weight; in fact, $x_{-\beta} \cdot v$ is nonzero for all nonzero $v$ in $M$.

Proof. See [9, Proposition 2.11].

Remark. Lemma 3.6 is not true for arbitrary Kac-Moody algebras as the following example shows.

Let

$$
A=\left[\begin{array}{rrr}
2 & -4 & 0 \\
-2 & 2 & -1 \\
0 & -1 & 2
\end{array}\right] \text {. }
$$

If $u^{t}=\left[\begin{array}{lll}7 & 8 & 3\end{array}\right]$ it is easily checked that $A u<0$ implying by [6, Corollary 4.3] that $A$ is a GCM of indefinite type. Since $D A$ is a symmetric matrix where $D=\operatorname{diag}\left(\frac{1}{2} 11\right)$, by [6, Theorem 9.11] the Kac-Moody algebra $\mathfrak{g}$ associated to $A$ is given by generators $\left\{e_{i}, f_{i}, i=1,2,3\right.$; with basis $\left.\alpha_{1}^{\mathrm{v}}, \alpha_{2}^{\mathrm{v}}, \alpha_{3}^{\mathrm{v}}\right\}$ and relations as given in $\S 1$, where $A=\left[a_{i_{j}}\right]$.

As $A$ is symmetrizable we have the standard invariant form on $\mathfrak{g}$ satisfying $\left(\alpha_{1}^{\mathrm{v}}, \alpha_{1}^{\mathrm{v}}\right)=4,\left(\alpha_{1}^{\mathrm{v}}, \alpha_{2}^{\mathrm{v}}\right)=-4,\left(\alpha_{1}^{\mathrm{v}}, \alpha_{3}^{\mathrm{v}}\right)=0,\left(\alpha_{2}^{\mathrm{v}}, \alpha_{3}^{\mathrm{v}}\right)=-1,\left(\alpha_{3}^{\mathrm{v}}, \alpha_{3}^{\mathrm{v}}\right)=2$, $\left(\alpha_{2}^{v}, \alpha_{2}^{v}\right)=2$. The canonical isomorphism $\nu: \mathfrak{h} \rightarrow \mathfrak{h}^{*}$ gives us $($,$) on \mathfrak{h}^{*}$ where $\left(\alpha_{1}, \alpha_{1}\right)=1,\left(\alpha_{1}, \alpha_{2}\right)=-2,\left(\alpha_{1}, \alpha_{3}\right)=0,\left(\alpha_{2}, \alpha_{2}\right)=2,\left(\alpha_{2}, \alpha_{3}\right)=-1$, $\left(\alpha_{3}, \alpha_{3}\right)=2$, where $\Pi=\left\{\alpha_{1}, \alpha_{2}, \alpha_{3}\right\}$ is the set of simple roots of $\mathfrak{g}$. We see that $\alpha_{1}+\alpha_{2}$ belongs to $\Delta_{+}$and that $\left(\alpha_{1}+\alpha_{2}, \alpha_{1}+\alpha_{2}\right)=-1$. By [6, Proposition 5.2], $\alpha_{1}+\alpha_{2}$ is thus imaginary.

Now let $M$ be the irreducible highest weight module over $\mathfrak{g}$ with highest weight $\Lambda$ in $\mathfrak{h}^{*}$ given by $\left\langle\Lambda, \alpha_{i}^{\mathrm{v}}\right\rangle=\delta_{i 3}$. It is easy to check that $M$ is faithful. Notice that since $\left\langle\Lambda, \alpha_{1}^{\mathrm{v}}\right\rangle=\left\langle\Lambda, \alpha_{2}^{\mathrm{v}}\right\rangle=0, f_{1} \cdot v^{+}=f_{2} \cdot v^{+}=0$ for any highest weight vector $v^{+}$in $M$. Thus $x_{-\left(\alpha_{1}+\alpha_{2}\right)} \cdot v^{+}=0$ for any nonzero $x_{-\left(\alpha_{1}+\alpha_{2}\right)}$ in $\mathfrak{g}_{-\left(\alpha_{1}+\alpha_{2}\right)}$.

Our next result, along with Lemma 3.3, gives us a criterion for determining the integrability or nonintegrability of a real root.

Lemma 3.7. If $\alpha$ belonging to $\Delta_{+}$has $\alpha_{k}$ in its support such that $\alpha_{k}$ belongs to $\Pi \backslash \Pi(\Lambda)$ and if $\lambda$ is a weight of $M$, then $\lambda-\alpha$ is also a weight. Thus if $\alpha$ is real it is nonintegrable.

Proof. Using Lemma 2.1 we write $\alpha=\alpha_{k}+\sum_{j=1}^{r} \alpha_{i_{j}}$ where $\alpha_{i_{j}}$ is simple for $j=1, \ldots, r$ and $\alpha_{k}+\sum_{j=1}^{t} \alpha_{i_{j}}$ is in $\Delta_{+}$for $t=1, \ldots, r$. Lemma 3.5 gives us the result when ht $\alpha=1$ so we assume that ht $\alpha>1$ and proceed by induction on ht $\alpha$. Assume that for any weight $\lambda, \lambda-\alpha_{k}-\sum_{j=1}^{r-1} \alpha_{i_{j}}$ is a weight of $M$.

If $\lambda-\alpha_{i_{r}}$ is a weight, the result is immediate so we consider the case when $\lambda-\alpha_{i_{r}}$ is not a weight of $M$. Notice then, that by Lemmas 3.5 and 3.1, $\alpha_{i_{r}}$ is integrable. It follows by Proposition 2.2 that $\lambda+t \alpha_{i_{r}}$ is a weight for all integers $t$ lying between 0 and $N$ where $N$ is a nonnegative integer satisfying 
$\left\langle\lambda, \alpha_{i_{r}}^{\mathrm{v}}\right\rangle=-N$. Proposition 2.2 also gives us nonnegative integers $p$ and $q$ such that $\alpha_{k}+\sum_{j=1}^{r-1} \alpha_{i_{j}}+s \alpha_{i_{r}}$ is a root for all integers $s$ between $-p$ and $q$ (note that $q$ is not 0 ), where

$$
p-q=\left\langle\alpha_{k}+\sum_{j=1}^{r-1} \alpha_{i_{j}}, \alpha_{i_{r}}^{\mathrm{v}}\right\rangle .
$$

Since $\operatorname{ht}\left(\alpha_{k}+\sum_{j=1}^{r-1} \alpha_{i_{j}}-s \alpha_{i_{r}}\right) \leq \mathrm{ht}\left(\alpha_{k}+\sum_{j=1}^{r-1} \alpha_{i_{j}}\right)$ for all $s$ between 0 and $p$ it follows that

$$
\lambda+t \alpha_{i_{r}}-\left(\alpha_{k}+\sum_{j=1}^{r-1} \alpha_{i_{j}}-s \alpha_{i_{r}}\right)
$$

is a weight when $s+t$ is between 0 and $N+p$. Since

$$
\left\langle\lambda-\left(\alpha_{k}+\sum_{j=1}^{r-1} \alpha_{i_{j}}\right), \alpha_{i_{r}}\right\rangle=-(N+p)+q,
$$

Proposition 2.2 again applies to give us that $\lambda-\left(\alpha_{k}+\sum_{j=1}^{r} \alpha_{i_{j}}\right)$ is a weight, as desired.

We see that in the event that $\mathfrak{g}$ is affine or finite dimensional and simple, we may readily classify a given positive root as integrable or nonintegrable. In general, however, the behavior of the imaginary roots under a given representation is not clearly understood. The following summarizes our results in terms of root strings.

Theorem 3.8. Let $\mathfrak{g}$ be a Kac-Moody algebra, $M$ a faithful irreducible highest weight representation of $\mathfrak{g}, \alpha$ a positive root of $\mathfrak{g}$, and $\lambda$ a weight of $M$.

(a) If $\mathfrak{g}$ is not indefinite, the following statements are equivalent:

(i) $\alpha$ is integrable;

(ii) the $\alpha$-string through $\lambda$ is finite;

(iii) $\alpha$ belongs to $\Delta_{+}(\Lambda) \cap \Delta^{\text {re }}$.

(b) Suppose $\mathfrak{g}$ is indefinite.

(i) If $\alpha$ is real, it is integrable if and only if it belongs to $\Delta_{+}(\Lambda)$.

(ii) If $\alpha$ does not belong to $\Delta_{+}(\Lambda)$, then the $\alpha$-string through $\lambda$ is infinite.

\section{BIBLIOGRAPHY}

1. I. B. Frenkel, Representations of Kac-Moody algebras and dual resonance models, Lectures in Appl. Math., vol. 21, Amer. Math. Soc., Providence, R.I., 1985, pp. 325-353.

2. __ Two constructions of affine Lie algebra representations and boson-fermion correspondence in quantum field theory, J. Funct. Anal. 44 (1981), 259-327.

3. I. B. Frenkel and V. G. Kac, Basic representations of affine Lie algebras and dual resonance models, Invent. Math. 62 (1980), 23-66. 
4. J. Humphreys, Introduction to Lie algebras and representation theory, 2nd ed., SpringerVerlag, Berlin, Heidelberg and New York, 1972.

5. N. Jacobson, Lie algebras, Interscience, New York, 1962.

6. V. G. Kac, Infinite dimensional Lie algebras, Birkhäuser, Boston, Mass., 1983.

7. _ Simple irreducible graded Lie algebras of finite growth, Math. USSR-Izv. 2 (1968), 1271-1311.

8. V. G. Kac, D. A. Kazhdan, J. Lepowsky and R. L. Wilson, Realizations of the basic representations of the Euclidean Lie algebras, Adv. in Math. 42 (1981), 83-112.

9. V. G. Kac and D. H. Peterson, Infinite dimensional Lie algebras, thet functions and modular forms, Adv. in Math. 53 (1984), 125-264.

10. J. Lepowsky, Some constructions of the affine Lie algebra $A_{1}^{(1)}$, Lectures in Appl. Math., vol. 21, Amer. Math. Soc., Providence, R. I., 1985, pp. 375-397.

11. __ Lectures on Kac-Moody algebras, Université Paris VI, spring 1978.

12. D. Mitzman, Integral bases for affine Lie algebras and their universal enveloping algebras, Contemp. Math., vol. 40, Amer. Math. Soc., Providence, R.I., 1985.

13. R. V. Moody, Euclidean Lie algebras, Canad. J. Math. 21 (1969), 1432-1454.

14. __ Lie algebras associated with generalized Cartan matrices, Bull. Amer. Math. Soc. $\mathbf{7 3}$ (1967), 217-221.

15. __ A new class of Lie algebras, J. Algebra 10 (1968), 211-230.

16. R. Steinberg, Lectures on Chevalley groups, Yale Univ. lecture notes, 1967.

Department of Mathematics and Computer Science, Indiana State University, Terre HaUte, INDiana 47809 